\title{
Sulfasalazine-Induced Pancytopenia in Ulcerative Colitis
}

\author{
N. S. Neki ${ }^{1}$, Ankur Jain ${ }^{2}$ \\ Received: September 28, 2015 Accepted: July 24, 2016 \\ doi: http://dx.doi.org/10.3329/jemc.v6i3.29685
}

\begin{abstract}
Sulfasalazine is a well-established disease-modifying agent. It is commonly used in the treatment of rheumatic disorders and inflammatory bowel disease. The most frequently reported adverse effects are gastrointestinal effects, headache, dizziness and rash; myelosuppression can also occur. Patients treated with sulfasalazine can develop thrombocytopenia which is immune mediated. We report a case of ulcerative colitis that was on sulfasalazine subsequently developing thrombocytopenia.
\end{abstract}

Key words: Sulfasalazine; Ulcerative colitis; Pancytopenia; Myelosuppression

J Enam Med Col 2016; 6(3): 164-165

\section{Introduction $^{1-4}$}

Ulcerative colitis (UC) is a chronic disease which is included in the category of inflammatory bowel disease along with Chrohn's disease. It is characterized by the inflammation and ulceration of the colonic mucosa. Sulfasalazine which is categorized under aminosalicylates is the first line medication for UC and it is a most effective drug for the induction of remission of $\mathrm{UC}$ as well as for the remission maintenance. It has been used in the treatment of inflammatory bowel disease for more than 40 years. It is less effective in Crohn's disease, where it exerts only a transient benefit in patients with active colonic disease and fails to prevent relapse or recurrence. Sulfasalazine is absorbed from the small intestine, re-excreted in bile and carried to the colon where its azo bond is split by bacteria to release sulfapyridine and 5-aminosalicylic acid. Sulfapyridine is absorbed and is responsible for most of the drug's side effects. 5-aminosalicylic acid is the active therapeutic moiety of the drug and exerts a beneficial topical action on the colonic mucosa. Side effects are common but are mainly reversible and these are related to high concentrations of sulfapyridine and to poor acetylation of the drug; they include gastrointestinal intolerance, malaise, headache, arthralgia, drug fever, effects on red blood cells and reversible male infertility. Rarely, neurotoxicity, hepatotoxicity, polyarteritis, pulmonary fibrosis, pancytopenia, a lupus-like syndrome and hemorrhagic colitis are produced. Pancytopenia in sulfasalazine occurs due to bone myelosuppression.

\section{Case report}

A 40-year-old male diagnosed as a case of ulcerative colitis at PGI Chandigarh was on continuous treatment with sulfasalazine $750 \mathrm{mg}$ thrice daily. He was also given prednisolone which was tapered off during his course of treatment. Patient presented to our medical emergency with the chief complaints of fever of 15 days duration associated with epistaxis of one day duration. $\mathrm{He}$ was hospitalized. His routine blood investigations revealed hemoglobin $6.5 \mathrm{gm} \%$, total leucocyte count $2456 / \mathrm{mm}^{3}$ and platelet count $32000 / \mathrm{mm}^{3}$. His thyroid function and serum B12 level were normal. Bone marrow examination could not be performed due to his refusal. A diagnosis of sulfasalazine-induced pancytopenia was made. Prednisolone $50 \mathrm{mg}$ once a day was started. He also received two units blood transfusion and one unit of single donor platelet concentrate. He was discharged within 10 days with hemoglobin of $9.5 \mathrm{gm} \%$, total leucocyte count $4200 / \mathrm{mm}^{3}$, and a platelet count of $78000 / \mathrm{mm}^{3}$. He was

1. Professor, Department of Medicine, Government Medical College/Guru Nanak Dev Hospital, Amritsar, India 2. Junior Resident, Department of Medicine, Government Medical College/Guru Nanak Dev Hospital, Amritsar, India Correspondence N. S. Neki, Email: drneki123@gmail.com 
advised to come for weekly follow-up. After one week he returned and his hemoglobin was found $10.2 \mathrm{gm} \%$, total leucocyte count $5400 / \mathrm{mm}^{3}$ and platelet count was $98600 / \mathrm{mm}^{3}$. At that time he was complaining of increased frequency of stools with blood staining. So he was given azathioprine $50 \mathrm{mg}$ twice daily along with prednisolone. Prednisolone was tapered off in 6 weeks. Patient is on regular follow-up with no complaints.

\section{Discussion}

Sulfasalazine is still a very important drug in the treatment of inflammatory bowel disease and rheumatoid or seronegative arthritis. Based on its cost and safety and convenience, many immunologists select sulfasalazine as initial treatment. The most common adverse effects are gastrointestinal effects, headache, dizziness and rash. It can also be associated with SLE like syndrome. ${ }^{5}$ Like numerous drugs, sulfasalazine can induce pancytopenia due to bone marrow toxicity. 6,7 Although bone marrow biopsy examinations could not be performed due to patient's refusal, on the basis of coincidence of changes in the hematological profile of the patient with changes in medication there was strong suggestion to bone marrow suppression and it was only due to sulfasalazine because he was on this drug. After stopping the drug, patient improved. When administered orally, $25 \%$ of sulfasalazine is absorbed in the small intestine and most of it reaches the colon where bacterial activity metabolizes it into two metabolites 5aminosalicylic acid and sulfapyridine. 5-aminosalicylic acid is not absorbed from the colon and manifests its therapeutic effects on the inflammatory mucosa. For this reason 5-aminosalicylic acid and sulfasalazine can be suitable alternatives and both have shown comparable efficacies. ${ }^{8}$ 5-aminosalicylic acid-induced pancytopenia suggestive of bone marrow suppression has also been reported. ${ }^{9}$ Sulfasalazine alone can also cause thrombocytopenia. Since there can be several mechanisms of bone marrow suppression such as immune reactions, infections and drug toxicity, it is recommended that sulfasalazine should be discontinued upon unexplained fever, chills, sore throat, malaise, or other nonspecific illness earlier in the treatment.

\section{Conclusion}

Sulfasalazine associated pancytopenia observed in the case reported herein is suspected due to bone marrow suppression. It is suggested that patient should be treated symptomatically and other causes of pancytopenia should be ruled out and drug should be stopped. Alternative drug to treat condition should be looked into.

\section{References}

1. Solberg IC, Lygren I, Jahnsen J. Clinical course during the first 10 years of ulcerative colitis: results from a populationbased inception cohort. Scand J Gastroenterol 2009; 44(4): 431-440.

2. Jani N, Regueiro MD. Medical therapy for ulcerative colitis. Gastroenterol Clin North Am 2002; 31(1): 147-166.

3. Plosker GL, Croom KF. Sulfasalazine: a review of its use in the management of rheumatoid arthritis. Pubmed 2005; 65(13): 1825-1849.

4. Maddocks JL, Slater DN. Toxic epidermal necrolysis, agranulocytosis and erythroid hypoplasia associated with sulphasalazine. J R Soc Med 1980; 73(8): 587-588.

5. Elliott MJ, Maini RN, Feldmann M, Kalden JR, Antoni C, Smolen JS et al. Treatment of rheumatoid arthritis with chimeric monoclonal antibodies to tumor necrosis factor- $\alpha$. Arthritis Rheum 1993; 36(12): 1681-1690.

6. Rhodes JM, Bartholomew TC, Jewell DP. Inhibition of leucocyte motility by drugs used in ulcerative colitis. Gut 1981; 22: 642-647.

7. Kim YC, Kim GM, Lee HS, Hong SI, Cheung DY, Kim JI et al. A case of hemophagocytic syndrome in an ulcerative colitis patient. Korean J Gastroenterol 2010; 56(1): 45-48.

8. Rachmilewitz D. Coated mesalazine (5-aminosalicylic acid) versus sulphasalazine in the treatment of active ulcerative colitis: a randomised trial. BMJ 1989; 298(6666): 82-86.

9. Kotanagi H, Ito M, Koyama K, Chiba M. Pancytopenia associated with 5-aminosalicylic acid use in a patient with Crohn's disease. J Gastroenterol 1998; 33(4): 571-574. 